\title{
Finite-Time Stabilization of Uncertain Switched Positive Linear Systems with Time-Varying Delays
}

\author{
Tianjian Yu, ${ }^{1}$ Yanke Zhong, ${ }^{2}$ Tefang Chen, ${ }^{2}$ and Chunyang Chen ${ }^{1}$ \\ ${ }^{1}$ School of Traffic and Transportation Engineering, Central South University, Changsha 410075, China \\ ${ }^{2}$ School of Information Science and Engineering, Central South University, Changsha 410075, China \\ Correspondence should be addressed to Yanke Zhong; zhongyanke1981@163.com
}

Received 30 September 2014; Revised 20 December 2014; Accepted 21 December 2014

Academic Editor: Muhammad Naveed Iqbal

Copyright (c) 2015 Tianjian Yu et al. This is an open access article distributed under the Creative Commons Attribution License, which permits unrestricted use, distribution, and reproduction in any medium, provided the original work is properly cited.

\begin{abstract}
This paper is concerned with finite-time stabilization (FTS) analysis for a class of uncertain switched positive linear systems with time-varying delays. First, a new definition of finite-time boundedness (FTB) is introduced for switched positive system. This definition can simplify FTS analysis. Taking interval and polytopic uncertainties into account, a robust state feedback controller is built such that the switched positive linear system is finite-time bounded. Finally, an example is employed to illustrate the validities of obtained results.
\end{abstract}

\section{Introduction}

As a special kind of hybrid dynamical systems, the switched system consists of finite subsystems and a switching law. The switching law orchestrates the switches between subsystems [1]. The switched positive system means that its output and state are nonnegative whenever the initial condition and input are nonnegative [2]. The switched positive systems are widely applied in practice, such as formation flying [3], communication system [4], viral mutation [5], and vehicle control [6].

In control theory, the stability and stabilization are basic problems for switched system. Many valuable results on these problems have been obtained, such as stability [7-12] and stabilization [13-15]. Most of the existing results focus on Lyapunov asymptotic stability (LAS). As we all know, LAS is defined over infinite-time interval. However, due to large state amplitude in transient process, some asymptotic stable systems may be useless [16]. Thus, FTB and FTS are introduced to analyze the transient performance of system. FTB implies that system state remains within a prescribed bound over a fixed interval. FTS means that designing a stabilization controller guarantees that the system is finitetime bounded. The main concern of FTB is the behavior of system state over a fixed finite time interval. It should be pointed out that there is not necessary relation between FTB and LAS. In practice, the finite-time bounded systems are employed in many fields, such as networked control systems [17] and network congestion control [18].

Since switched system contains several dynamic subsystems and a switching law, it is difficult to analyze FTB and FTS. Recently, these problems have been extensively studied. Based on the average dwell-time approach, Lin et al. investigated FTB for switched systems with fixed delays [19]. However, the time delay is always time-varying in practice. Then the application of obtained results is limited. Taking time-varying delay into account, M. Xiang and Z. Xiang analyzed FTB and FTS for switched linear systems [20]. In the above-mentioned literatures, the switching instants must be scheduled in advance. Therefore the corresponding switching law is time-dependent. However, the switching instants are determined by system state in some switched system. In this case, the obtained approaches may be useless. In [21], a statedependent switching law was designed such that the switched linear system was finite-time bounded. In order to simplify the mathematical calculation, Zhong and Chen adopted a new method to analyze the FTB [22]. In fact, the switches between subsystems produce great effect on system state. Thus, for the problems of FTB and FTS, it is significant to investigate this issue. In [23], Xiang and Xiao investigated the switching behavior's impacts on system state. Furthermore, a state feedback controller was built such that the system was 
finite-time bounded. In practice, the researchers sometimes care about not only whether the system could be stabilized but also whether the stabilization time meets the requirements. In [24], some criteria were proposed on designing an optimal switching law. Naveed et al. investigated the problem of finitetime $\mathrm{H}$-infinity state estimation for switched systems under asynchronous switching [25], and the developed method is useful for FTB and FTS. There are some other latest literatures [26-29] related to FTB and FTS, and the references are cited therein.

About the mentioned literatures, we need to pay attention to the following facts. First, switched positive system is an important kind of switched system. However, there are few works on its FTB and FTS. Second, most of the obtained results are based on a common assumption that the norm of external disturbance is bounded over infinite time interval. But the external disturbance usually does not satisfy this strict assumption. In this case, the obtained results may be unsuitable for application. Thus, it is very significant to reduce the restriction on external disturbance. Besides, the definition of FTB contains a product term of gain matrix and state vector; therefore complex mathematical calculations are unavoidable. Finally, the state of switched positive system is nonnegative. Then, whether a more concise definition of FTB could be proposed for switched positive system to simplify the analysis of FTS is a problem that naturally arises.

Motivated by the above considerations, we investigate FTS for uncertain switched positive linear systems. The main object of this paper is to design a robust state feedback controller such that the system is finite-time bounded. The main contributions and novelties of this paper are summarized as follows: (1) for switched positive system, a much more concise definition of FTB is introduced to simplify mathematical calculation; (2) a state feedback controller is built for the uncertain switched positive linear system with time-varying delay; (3) different from most of other results, the disturbance is only required to be bounded in this paper; (4) taking the interval and polytopic uncertainties into account, the obtained controller is robust to uncertainties.

The remainder of the paper is organized as follows. In Section 2, some necessary definitions and lemmas are introduced. In Section 3, a robust stabilization controller is designed for uncertain switched positive linear systems with time-varying delays. A numerical example is given in Section 4 . The paper is concluded by Section 5 .

Notation. $R^{n}\left(R_{+}^{n}\right)$ denotes the $n$-dimensional real (positive) vector space; $M$ represents Metzler matrices whose offdiagonal entries are nonnegative; $A>0(A \succeq 0, A \prec 0)$ means that all elements of matrix $A$ are positive (nonnegative, negative); let $\underline{m}=[1, \ldots, m]$, where $m$ is positive integer.

\section{Preliminaries and Problem Formulation}

Consider a switched linear system described as

$$
\begin{aligned}
\dot{x}(t)= & \left(A_{\sigma(t)}+\Delta A_{\sigma(t)}\right) x(t) \\
& +\left(B_{\sigma(t)}+\Delta B_{\sigma(t)}\right) x(t-h(t))
\end{aligned}
$$

$$
\begin{array}{r}
+F_{\sigma(t)} u(t)+G_{\sigma(t)} w(t) \\
0 \leq h(t) \leq d, \quad 0 \leq t, \\
x(t)=\varphi(t) \quad t \in[-d, 0),
\end{array}
$$

where $x(t) \in R^{n}$ represents the system state; $\sigma(t)$ is the switching law which is a piecewise continuous function with $\sigma(t) \in \underline{m}$; the switched system is composed of $m$ subsystems; if $\sigma(t)=i$, the $i$ th subsystem is activated; $A_{\sigma(t)}, B_{\sigma(t)}, F_{\sigma(t)}$, and $G_{\sigma(t)} \geq 0$ are known constant matrices with appropriate dimension; $\Delta A_{\sigma(t)}$ and $\Delta B_{\sigma(t)}$ represent uncertainties satisfying $\left(B_{\sigma(t)}+\Delta B_{\sigma(t)}\right) \geq 0 ; h(t)$ represents the time-varying delay; $u(t)$ is the control input; $w(t)$ denotes the external disturbance; $\varphi(t) \geq 0$ is the continuous vector-valued initial function over $t \in[-d, 0)$; and $d$ is a positive constant.

The state feedback controller is given as follows:

$$
u(t)=H_{\sigma(t)} x(t)
$$

where $H_{\sigma(t)}$ is the gain matrix to be determined.

The main task is to choose appropriate $H_{\sigma(t)}$ such that system (1) is finite-time bounded.

Define

$$
\begin{gathered}
\widetilde{A}_{\sigma(t)}=\left(A_{\sigma(t)}+\Delta A_{\sigma(t)}\right), \\
\widetilde{B}_{\sigma(t)}=\left(B_{\sigma(t)}+\Delta B_{\sigma(t)}\right) .
\end{gathered}
$$

Applying (2) to (1), the following closed-loop system is obtained:

$$
\begin{gathered}
\dot{x}(t)=\left(\widetilde{A}_{\sigma(t)}+F_{\sigma(t)} H_{\sigma(t)}\right) x(t) \\
+\widetilde{B}_{\sigma(t)} x(t-h(t))+G_{\sigma(t)} w(t) \\
0 \leq h(t) \leq d, \quad 0 \leq t, \\
x(t)=\varphi(t) \quad t \in[-d, 0) .
\end{gathered}
$$
tions.

Assume that system (1) satisfies the following assumpAssumption 1 (see [30]). The external disturbance $w(t)$ satisfies

$$
0 \preceq w(t) \preceq \gamma .
$$

Remark 2. In most of the existing literatures, the external disturbance should satisfy

$$
\int_{0}^{\infty} \omega^{T}(t) \omega(t) d t \leq \theta \quad 0<\theta .
$$

Obviously, condition (6) is much stricter than (5). In Assumption 1, the external disturbance is only required to be bounded. In addition, Assumption 1 conforms much more to the practical situation.

Assumption 3. The time-varying delay satisfies

$$
\dot{h}(t) \leq \rho, \quad 0 \leq h(t) \leq d, \quad \rho \leq 1 .
$$


Assumption 4. The state trajectory is continuous everywhere. Furthermore, the switching number of $\sigma(t)$ is finite over fixed finite-time interval.

Next, Lemma 5 is introduced for subsequent analysis.

Lemma 5. System (3) is positive if and only if $\left(\widetilde{A}_{\sigma(t)}+\right.$ $\left.F_{\sigma(t)} H_{\sigma(t)}\right) \in M, \widetilde{B}_{\sigma(t)} \geq 0, G_{\sigma(t)} \geq 0$, and initial states $x_{0} \geq 0$ and $w(t) \geq 0$ hold for $\sigma(t) \in \underline{m}[26]$.

Definition 6 is proposed as follows.

Definition 6 (see [31]). Forgiven positive constants $C_{1}<C_{2}$, $T_{f}, \gamma$, and a switching signal $\sigma(t)$, if

$$
\left\|x\left(t_{0}\right)\right\| \leq C_{1} \Longrightarrow\|x(t)\|<C_{2} \quad \forall t \in\left[0, T_{f}\right], \forall w(s) \preceq \gamma,
$$

then system (3) is said to be finite-time bounded with respect to $\left(C_{1}, C_{2}, T_{f}, \gamma, \sigma(t)\right)$, where $\|x\|=\sum_{i=1}^{n}\left|x_{i}\right| ; x_{i}$ is the $i$ th element of vector $x$. In this paper, we specify $C_{1}=$ $\sup _{t \in[-d, 0)}\|x(t)\|$.

Remark 7. According to the definition of FTB in most of other literatures, system (3) should satisfy

$$
x^{T}\left(t_{0}\right) R x\left(t_{0}\right) \leq C_{1} \Longrightarrow x^{T}(t) R x(t)<C_{2} \quad \forall t \in\left[0, T_{f}\right] .
$$

Note the differences between (8) and (9). First, compared with (9), the gain matrix $R$ is removed from (8). This improvement brings in much convenience for subsequence process. Some complex matrix transformations could be avoided. Besides, if we choose the definition (9), it is not convenient to adopt multiply copositive Lyapunov-Krasovskii function to analyze FTB due to the term of $x^{T}(t) x(t)$. Therefore, $x^{T}(t) x(t)$ is replaced by $\|x(t)\|$ which can simplify subsequent mathematical analysis.

Remark 8. The essence of FTB lies in that the system state must remain within the prescribed bound over fixed interval. Since both (8) and (9) can guarantee it, the two different definitions are consistent in essence.

Definition 9. For $T \geq t \geq 0$, let $N_{\sigma(t)}(t, T)$ denote the switching number of $\sigma(t)$ over $(t, T]$. If

$$
N_{\sigma(t)}(t, T) \leq N_{0}+\frac{T-t}{\tau_{a}}
$$

holds for $\tau_{a} \geq 0$ and an integer $N_{0} \geq 0$, then $\tau_{a}$ is called an average dwell-time [16].

\section{Main Result}

Assume that the uncertainties in system (3) are interval or polytopic uncertainties. First, system (3) with interval uncertainties is considered. In this case, we build a robust state feedback controller such that system (3) is finite-time bounded.
Theorem 10. Assume that $\underline{A}_{i} \preceq \widetilde{A}_{i} \preceq \bar{A}_{i}, 0 \preceq \underline{B}_{i} \preceq \widetilde{B}_{i} \preceq \bar{B}_{i}$ for $i \in \underline{m} . \underline{A}_{i}, \bar{A}_{i}, \underline{B}_{i}$, and $\bar{B}_{i}$ are known matrices. For given positive constants $C_{1}, C_{2}, T_{f}, \gamma, \lambda$, and $\beta>1$, if there exist vectors $v_{i} \geq 0, \eta_{i} \geq 0, \zeta_{i} \geq 0$, and $\xi_{i}$ such that

$$
\begin{gathered}
\operatorname{diag}\left\{\psi_{1}, \ldots, \psi_{n}, \phi_{1}, \ldots, \phi_{n}\right\}<0, \\
v_{i} \leq \beta v_{j}, \quad \eta_{i} \leq \beta \eta_{j}, \quad \zeta_{i} \leq \beta \zeta_{j} \\
\text { for } i, j \in[1,2, \ldots, m], \\
\left(\underline{A}_{i}+F_{i} H_{i}\right)_{l, p}>0 \quad l, p \in \underline{n}, \quad l \neq p,
\end{gathered}
$$

and the average dwell-time satisfies

$$
\tau_{a}>\frac{T_{f} \ln \beta}{\ln C_{2}+\ln K_{4}-\ln \left(\Phi_{1}+\Phi_{2}\right)},
$$

then under the controller $u(t)=H_{\sigma(t)} x(t)$ with gain matrix $H_{\sigma(t)}$ satisfying

$$
\xi_{i}=\left(F_{i} H_{i}\right)^{T} v_{i}
$$

system (3) is finite-time bounded with respect to $\left(C_{1}, C_{2}, T_{f}\right.$, $\gamma, \sigma(t))$ under the switching law (14).

$$
\psi_{j}=\left(\bar{A}_{i}^{T}\right)_{j} v_{i}+\xi_{i, j}-\lambda v_{i, j}+\eta_{i, j}+d \zeta_{i, j}, \phi_{j}=\left(\bar{B}_{i}^{T}\right)_{j} v_{i}-(1-
$$
$\rho) \eta_{i, j}, i \in \underline{m}, j \in \underline{n} ;\left(\bar{A}_{i}^{T}\right)_{j}$ denotes the jth row of matrix $\bar{A}_{i}^{T}$; $\xi_{i, j}, v_{i, j}, \eta_{i, j}$, and $\zeta_{i, j}$ represent the $j$ th element of vectors $\xi_{i}, v_{i}, \eta_{i}$, and $\zeta_{i}$, respectively; $\left(\underline{A}_{i}+F_{i} H_{i}\right)_{l, p}$ is the lth row and pth column element of matrix $\left(\underline{A}_{i}+F_{i} H_{i}\right) ; K_{0}=\max _{i \in m} \lambda\left(G_{i}\right)$, and $\lambda\left(G_{i}\right)$ denotes all eigenvalues of matrix $G_{i} ; K_{1}=\max \left(v_{i, j}\right), K_{2}=$ $\max \left(\eta_{i, j}\right), K_{3}=\max \left(\zeta_{i, j}\right)$, and $K_{4}=\min \left(v_{i, j}\right)$ for $i \in \underline{m}, j \in \underline{n}$. In addition, $\Phi_{1}=\left(K_{1}+d e^{\lambda d} K_{2}+d^{2} e^{\lambda d} K_{3}\right) \sup _{t \in[-d, 0)}\|x(t)\|$; $\Phi_{2}=e^{2 \lambda T_{f}} T_{f} n \gamma K_{0} K_{1}$.

Remark 11. Due to the existence of interval uncertainties, system matrices $\widetilde{A}_{i}$ and $\widetilde{B}_{i}$ are unknown matrices. However, their value ranges usually are known in practice. In fact, $\bar{A}_{i}$ and $\bar{B}_{i}$ denote the upper bounds of $\widetilde{A}_{i}$ and $\widetilde{B}_{i}$, respectively; $\underline{A}_{i}$ and $\underline{B}_{i}$ are the lower bounds of $\widetilde{A}_{i}$ and $\widetilde{B}_{i}$, respectively.

Proof. Since (13) holds, $\left(\underline{A}_{i}+F_{i} H_{i}\right) \in M$. Noting that $\underline{A}_{i} \preceq \widetilde{A_{i}}$, then $\left(\widetilde{A}_{i}+F_{i} H_{i}\right) \in M$. Besides, $\widetilde{B}_{i} \geq 0, G_{i} \geq 0, \varphi(t) \geq 0$, and $0 \preceq w(t)$. By Lemma 5, system (3) is a switched positive system.

Construct multiply copositive Lyapunov-Krasovskii function for system (3) as follows:

$$
V(t)=V_{i}(t)=V_{i, 1}(t)+V_{i, 2}(t)+V_{i, 3}(t),
$$

where

$$
\begin{gathered}
V_{i, 1}(t)=x^{T}(t) v_{i}, \\
V_{i, 2}(t)=\int_{t-h(t)}^{t} e^{\lambda(t-s)} x^{T}(s) \eta_{i} d s \\
V_{i, 3}(t)=\int_{-d}^{0} \int_{t+\theta}^{t} e^{\lambda(t-s)} x^{T}(s) \zeta_{i} d s d \theta .
\end{gathered}
$$


Taking the derivatives of $V_{i, 1}(t), V_{i, 2}(t)$, and $V_{i, 3}(t)$ with respect to $t$ along the trajectory of system (3) on $t \in\left[t_{k}, t_{k+1}\right)$, we have

$$
\begin{aligned}
\dot{V}_{i, 1}(t)= & \lambda V_{i, 1}(t)+x^{T}(t)\left(\widetilde{A}_{i}+F_{i} H_{i}\right)^{T} v_{i} \\
& +x^{T}(t-h(t)) \widetilde{B}_{i}^{T} v_{i}+w^{T}(t) G_{i}^{T} v_{i}-\lambda x^{T}(t) v_{i} \\
\leq & \lambda V_{i, 1}(t)+x^{T}(t)\left(\bar{A}_{i}+F_{i} H_{i}\right)^{T} v_{i} \\
& +x^{T}(t-h(t)) \bar{B}_{i}^{T} v_{i}+w^{T}(t) G_{i}^{T} v_{i}-\lambda x^{T}(t) v_{i}, \\
\dot{V}_{i, 2}(t)= & \lambda V_{i, 2}(t)+x^{T}(t) \eta_{i}-(1-\dot{h}(t)) e^{\lambda h(t)} x^{T}(t-h(t)) \eta_{i} \\
\leq & \lambda V_{i, 2}(t)+x^{T}(t) \eta_{i}-(1-\rho) x^{T}(t-h(t)) \eta_{i}, \\
\dot{V}_{i, 3}(t)= & \lambda V_{i, 3}(t)+d x^{T}(t) \zeta_{i}-\int_{t-d}^{t} e^{\lambda(t-s)} x^{T}(s) \zeta_{i} d s \\
\leq & \lambda V_{i, 3}(t)+d x^{T}(t) \zeta_{i}-\int_{t-d}^{t} x^{T}(s) \zeta_{i} d s .
\end{aligned}
$$

From (18), it follows that

$$
\begin{aligned}
\dot{V}_{i}(t)-\lambda V_{i}(t) \leq & x^{T}(t)\left(\left(\bar{A}_{i}+F_{i} H_{i}\right)^{T} v_{i}-\lambda v_{i}+\eta_{i}+d \zeta_{i}\right) \\
& +x^{T}(t-h(t))\left(\bar{B}_{i}^{T} v_{i}-(1-\rho) \eta_{i}\right) \\
& +w^{T}(t) G_{i}^{T} v_{i}-\int_{t-d}^{t} x^{T}(s) \zeta_{i} d s .
\end{aligned}
$$

It is derived from (11) that

$$
\begin{gathered}
x^{T}(t)\left[\left(\bar{A}_{i}+F_{i} H_{i}\right)^{T} v_{i}-\lambda v_{i}+\eta_{i}+d \zeta_{i}\right]<0, \\
x^{T}(t-h(t))\left[\bar{B}_{i}^{T} v_{i}-(1-\rho) \eta_{i}\right]<0 .
\end{gathered}
$$

Applying (20) to (19), we get

$$
\dot{V}_{i}(t)-\lambda V_{i}(t)<w^{T}(t) G_{i}^{T} v_{i} .
$$

Since $(d / d t)\left(e^{-\lambda t} V_{i}(t)\right)=e^{-\lambda t}\left[\dot{V}_{i}(t)-\lambda V_{i}(t)\right]$, (21) is transformed into

$$
\frac{d}{d t}\left(e^{-\lambda t} V_{i}(t)\right)<e^{-\lambda t} w^{T}(t) G_{i}^{T} v_{i} .
$$

Let $t_{k}$ stand for the instant of the $K$ th switching and $t_{k^{-}}$ denote the instant just before $t_{k}$. Integrating from $t_{k}$ to $t$ on both sides of (22), then

$$
V_{i}(t)<e^{\lambda\left(t-t_{k}\right)} V_{i}\left(t_{k}\right)+\int_{t_{k}}^{t} w^{T}(s) e^{\lambda(t-s)} G_{i}^{T} v_{i} d s .
$$

Noting (12) and the continuity of $x(t)$, then

$$
\begin{gathered}
V_{i}(t)<\beta e^{\lambda\left(t-t_{k}\right)} V_{i}\left(t_{k^{-}}\right)+\int_{t_{k}}^{t} w^{T}(s) e^{\lambda(t-s)} G_{i}^{T} v_{i} d s, \\
V_{i}\left(t_{k^{-}}\right)<e^{\lambda\left(t_{t}-t_{k-1}\right)} V_{i}\left(t_{k-1}\right)+\int_{t_{k-1}}^{t_{k}} w^{T}(s) e^{\lambda(t-s)} G_{i}^{T} v_{i} d s .
\end{gathered}
$$

It is obtained via (24) that

$$
\begin{aligned}
V_{i}(t)< & \beta^{2} e^{\lambda\left(t-t_{k-1}\right)} V_{i}\left(t_{(k-1)^{-}}\right) \\
& +\beta e^{\lambda\left(t-t_{k}\right)} \int_{t_{k-1}}^{t_{k}} w^{T}(s) e^{\lambda(t-s)} G_{i}^{T} v_{i} d s \\
& +\int_{t_{k}}^{t} w^{T}(s) e^{\lambda(t-s)} G_{i}^{T} v_{i} d s .
\end{aligned}
$$

Assume that the switching number of $\sigma(t)$ over $\left[0, T_{f}\right]$ is $N$. We get

$$
\begin{aligned}
V_{i}(t)< & \beta^{N} e^{\lambda t} V_{i}(0) \\
& +\beta^{N} e^{\lambda\left(t-t_{1}\right)} \int_{t_{0}}^{t_{1}} w^{T}(s) e^{\lambda(t-s)} G_{i}^{T} v_{i} d s \\
& +\cdots+\beta e^{\lambda\left(t-t_{k}\right)} \int_{t_{k-1}}^{t_{k}} w^{T}(s) e^{\lambda(t-s)} G_{i}^{T} v_{i} d s \\
& +\int_{t_{k}}^{t} w^{T}(s) e^{\lambda(t-s)} G_{i}^{T} v_{i} d s \\
< & \beta^{N} e^{\lambda T_{f}} V_{i}(0)+\beta^{N} e^{\lambda T_{f}} \int_{t_{0}}^{t_{1}} w^{T}(s) e^{\lambda(t-s)} G_{i}^{T} v_{i} d s \\
& +\cdots+\beta^{N} e^{\lambda T_{f}} \int_{t_{k-1}}^{t_{k}} w^{T}(s) e^{\lambda(t-s)} G_{i}^{T} v_{i} d s \\
& +\beta^{N} e^{\lambda T_{f}} \int_{t_{k}}^{t} w^{T}(s) e^{\lambda(t-s)} G_{i}^{T} v_{i} d s \\
= & \beta^{N} e^{\lambda T_{f}} V_{i}(0)+\beta^{N} e^{\lambda T_{f}} \int_{t_{0}}^{t} w^{T}(s) e^{\lambda(t-s)} G_{i}^{T} v_{i} d s \\
< & \beta^{N} e^{\lambda T_{f}} V_{i}(0)+\beta^{N} e^{2 \lambda T_{f}} K_{0} \int_{t_{0}}^{t} w^{T}(s) v_{i} d s \\
< & \beta^{N} e^{\lambda T_{f}} V_{i}(0)+\beta^{N} e^{2 \lambda T_{f}} K_{0} \int_{t_{0}}^{t} n \gamma K_{1} d s \\
= & \beta^{N} e^{\lambda T_{f}} V_{i}(0)+\beta^{N} e^{2 \lambda T_{f}} T_{f} n \gamma K_{0} K_{1} .
\end{aligned}
$$

According to the definition of $V_{i}(0)$,

$$
\begin{aligned}
V(0)= & x^{T}(0) v_{\sigma(0)}+\int_{-h(0)}^{0} e^{-\lambda s} x^{T}(s) \eta_{\sigma(0)} d s \\
& +\int_{-d}^{0} \int_{\theta}^{0} e^{-\lambda s} x^{T}(s) \zeta_{\sigma(0)} d s d \theta \\
\leq & x^{T}(0)\left[K_{1}^{T}, \ldots, K_{1}^{T}\right]^{T} \\
& +\int_{-d}^{0} e^{-\lambda s} x^{T}(s)\left[K_{2}^{T}, \ldots, K_{2}^{T}\right]^{T} d s \\
& +\int_{-d}^{0} \int_{\theta}^{0} e^{-\lambda s} x^{T}(s)\left[K_{3}^{T}, \ldots, K_{3}^{T}\right]^{T} d s d \theta
\end{aligned}
$$




$$
\begin{aligned}
\leq & x^{T}(0)\left[K_{1}^{T}, \ldots, K_{1}^{T}\right]^{T} \\
& +e^{\lambda d} \int_{-d}^{0} x^{T}(s)\left[K_{2}^{T}, \ldots, K_{2}^{T}\right]^{T} d s \\
& +e^{\lambda d} \int_{-d}^{0} \int_{\theta}^{0} x^{T}(s)\left[K_{3}^{T}, \ldots, K_{3}^{T}\right]^{T} d s d \theta \\
\leq & K_{1}\|x(0)\|+d e^{\lambda d} K_{2} \sup _{t \in[-d, 0]}\|x(t)\| \\
& +d^{2} e^{\lambda d} K_{3} \sup _{t \in[-d, 0]}\|x(t)\| \\
\leq & \left(K_{1}+d e^{\lambda d} K_{2}+d^{2} e^{\lambda d} K_{3}\right) \sup _{t \in[-d, 0]}\|x(t)\| .
\end{aligned}
$$

Substituting (27) into (26) yields

$$
\begin{aligned}
V_{i}(t)< & \beta^{N} e^{\lambda T_{f}}\left(K_{1}+d e^{\lambda d} K_{2}+d^{2} e^{\lambda d} K_{3}\right) \sup _{t \in[-d, 0]}\|x(t)\| \\
& +\beta^{N} e^{2 \lambda T_{f}} T_{f} n \gamma K_{0} K_{1} .
\end{aligned}
$$

Obviously,

$$
x^{T}(t)\left[K_{4}^{T}, \ldots, K_{4}^{T}\right]^{T}<x^{T}(t) v_{i}<V_{i}(t)
$$

It is derived from (28) and (29) that

$\|x(t)\|$

$$
\begin{aligned}
& <\left(\beta^{N} e^{\lambda T_{f}}\left(K_{1}+d e^{\lambda d} K_{2}+d^{2} e^{\lambda d} K_{3}\right) \sup _{t \in[-d, 0]}\|x(t)\|\right. \\
& \left.\quad+\beta^{N} e^{2 \lambda T_{f}} T_{f} n \gamma K_{0} K_{1}\right) \\
& \quad \times\left(K_{4}\right)^{-1} \\
& =\frac{\beta^{N}\left(\Phi_{1}+\Phi_{2}\right)}{K_{4}} .
\end{aligned}
$$

On the other hand, (31) could be derived from (14):

$$
\begin{gathered}
\frac{T_{f} \ln \beta}{\ln C_{2}+\ln K_{4}-\ln \left(\Phi_{1}+\Phi_{2}\right)}<\tau_{a}, \\
\frac{T_{f}}{\tau_{a}}<\frac{\ln C_{2}+\ln K_{4}-\ln \left(\Phi_{1}+\Phi_{2}\right)}{\ln \beta}, \\
N<\frac{\ln C_{2}+\ln K_{4}-\ln \left(\Phi_{1}+\Phi_{2}\right)}{\ln \beta}, \\
N \ln \beta<\ln \frac{C_{2} K_{4}}{\Phi_{1}+\Phi_{2}}, \\
\beta^{N} \frac{\Phi_{1}+\Phi_{24}}{K_{4}}<C_{2} .
\end{gathered}
$$

Consequently, $\|x(t)\|<C_{2}$.
According to Definition 6, the system (3) is finite-time bounded. Thus, the designed controller is the desired controller. This completes the proof of Theorem 10.

Remark 12. Note (11) and (15). If the $\xi_{i}$ in (11) is replaced by $\left(F_{i} H_{i}\right)^{T} v_{i}$, then the inequality (11) is not LMIS. In this case, we could not solve (11) via MATLAB. Thus, we should analyze (11) directly. Note that $\psi_{j}=\left(\bar{A}_{i}^{T}\right)_{j} v_{i}+\xi_{i, j}-\lambda v_{i, j}+\eta_{i, j}+d \zeta_{i, j}$ and $\phi_{j}=\left(\bar{B}_{i}^{T}\right)_{j} v_{i}-(1-\rho) \eta_{i, j} \cdot\left(\bar{A}_{i}^{T}\right)_{j},\left(\bar{B}_{i}^{T}\right)_{j}, \lambda$, and $d$ are known; $v_{i}, \xi_{i, j}, v_{i, j}, \eta_{i, j}$, and $\zeta_{i, j}$ are unknown. We find that $\psi_{j}$ and $\phi_{j}$ do not contain any product term between two unknown variables. Therefore inequality (11) is LMIS. $\xi_{i}$ and $v_{i}$ could have been obtained by solving (11). Then substitute them into (15). In this way, $H_{i}$ is obtained finally.

Remark 13. Compared with other literatures, such as $[19,21$, 22], the derivation process of Theorem 10 in this paper is much more concise. The main reason lies in that the definition on FTB in this paper is much more concise compared with other literatures, such as [19, 21, 22]. Thus complex matrix translations are eliminated. Besides, the description on FTB is also very concise in [20, see Definition 5]. However, the physical meaning of Definition 6 in this paper is much clearer.

Next, the attention is focused on designing a state feedback controller such that the system (3) with polytopic uncertainties is finite-time bounded.

Theorem 14. Assume that $\Gamma_{i}=\left\{\widetilde{A}_{i}^{p}, p \in \underline{n}\right\}$ and $\Theta_{i}=\left\{\widetilde{B}_{i}^{p}, p \in\right.$ $\underline{n}\}, i \in \underline{m} ; \Phi_{i}$ and $\Theta_{i}$ denote the convex hull of the vertices $\widetilde{A}_{i}^{p}$ and $\widetilde{B}_{i}^{p}$, respectively. $\widetilde{A}_{i}^{p}$ and $\widetilde{B}_{i}^{p} \geq 0$ are known matrices. For given positive constants $C_{1}, C_{2}, T_{f}, \gamma, \lambda$, and $\beta>1$, if there exist vectors $v_{i} \geq 0, \eta_{i} \geq 0, \zeta_{i} \geq 0$, and $\xi_{i}$ such that

$$
\begin{gathered}
\operatorname{diag}\left\{\psi_{1}, \ldots, \psi_{n}, \phi_{1}, \ldots, \phi_{n}\right\}<0, \\
v_{i} \leq \beta v_{j}, \quad \eta_{i} \leq \beta \eta_{j}, \quad \zeta_{i} \leq \beta \zeta_{j}, \\
\text { for } i, j \in[1,2, \ldots, m], \\
\left(\widetilde{A}_{i}^{p}+F_{i} H_{i}\right)_{l, j}>0 \quad l, j \in \underline{n}, l \neq j,
\end{gathered}
$$

and the average dwell-time satisfies

$$
\tau_{a}>\frac{T_{f} \ln \beta}{\ln C_{2}+\ln K_{4}-\ln \left(\Phi_{1}+\Phi_{2}\right)},
$$

then under the controller $u(t)=H_{\sigma(t)} x(t)$ with gain matrix $H_{\sigma(t)}$ satisfying

$$
\xi_{i}=\left(F_{i} H_{i}\right)^{T} v_{i}
$$

system (3) is finite-time bounded with respect to $\left(C_{1}, C_{2}, T_{f}\right.$, $\gamma, \sigma(t))$ under the switching law (35).

$$
\psi_{j}=\left(\widetilde{A}_{i}^{p}\right)_{j}^{T} v_{i}+\xi_{i, j}-\lambda v_{i, j}+\eta_{i, j}+d \zeta_{i, j}, \phi_{j}=\left(\widetilde{B}_{i}^{p}\right)_{j}^{T} v_{i}-(1-
$$
$\rho) \eta_{i, j}, i \in \underline{m}, j \in \underline{n} ;\left(\widetilde{A}_{i}^{p}\right)_{j}^{T}$ denotes the jth row of matrix $\left(\widetilde{A}_{i}^{p}\right)^{T}$; $v_{i, j}, \eta_{i, j}$, and $\zeta_{i, j}$ represent the $j$ th element of vectors $v_{i}, \eta_{i}$, and $\zeta_{i}$, respectively; $\left(\widetilde{A}_{i}^{p}+F_{i} H_{i}\right)_{l, p}$ is the lth row and pth column 
element of matrix $\left(\widetilde{A}_{i}^{p}+F_{i} H_{i}\right)$; let $K_{0}=\max _{i \in m} \lambda\left(G_{i}\right) ; \lambda\left(G_{i}\right)$ denotes all eigenvalues of matrix $G_{i} ; K_{1}=\max \left(v_{i, j}\right), K_{2}=$ $\max \left(\eta_{i, j}\right), K_{3}=\max \left(\zeta_{i, j}\right)$, and $K_{4}=\min \left(v_{i, j}\right)$ for $i \in \underline{m}, j \in \underline{n} ;$ In addition, $\Phi_{1}=\left(K_{1}+d e^{\lambda d} K_{2}+d^{2} e^{\lambda d} K_{3}\right) \sup _{t \in[-d, 0)}\|x(t)\|$; $\Phi_{2}=e^{2 \lambda T_{f}} T_{f} n \gamma K_{0} K_{1}$.

Proof. By the property of convex hull, $\widetilde{A}_{i}=\sum_{p=1}^{n} \gamma_{p} \widetilde{A}_{i}^{p}$, $0<\gamma_{p}<1$, and $\sum_{p=1}^{n} \gamma_{p}=1$ for $i \in \underline{m}$. Furthermore, $\left(\widetilde{A}_{i}^{p}+F_{i} H_{i}\right)_{l, j}>0 l, j \in \underline{n}$ and $l \neq j$; therefore $\left(\sum_{p=1}^{n} \gamma_{p} \widetilde{A}_{i}^{p}+\right.$ $\left.\sum_{p=1}^{n} \gamma_{p} F_{i} H_{i}\right)_{l, j}>0$ which implies that $\left(\widetilde{A_{i}}+F_{i} H_{i}\right) \in M$. Besides, $\widetilde{B}_{i} \geq 0, G_{i} \geq 0, \varphi(t) \geq 0$ and $0 \preceq w(t)$. By Lemma 5 , system (3) is a switched positive system.

Construct multiply copositive Lyapunov-Krasovskii functions which have the same structure of (16). Taking the derivatives of $V_{i, 1}(t), V_{i, 2}(t)$, and $V_{i, 3}(t)$ with respect to $t$ along the trajectory of system (3) on $t \in\left[t_{k}, t_{k+1}\right)$, we get

$$
\begin{aligned}
\dot{V}_{i}(t)-\lambda V_{i}(t) \leq & x^{T}(t)\left(\left(\widetilde{A}_{i}+F_{i} H_{i}\right)^{T} v_{i}-\lambda v_{i}+\eta_{i}+d \zeta_{i}\right) \\
& +x^{T}(t-h(t))\left(\widetilde{B}_{i} v_{i}-(1-\rho) \eta_{i}\right) \\
& +w^{T}(t) G_{i}^{T} v_{i}-\int_{t-d}^{t} x^{T}(s) \zeta_{i} d s .
\end{aligned}
$$

It is derived from (32) that

$$
\left(\gamma_{p} \widetilde{A}_{i}^{p}+\gamma_{p} F_{i} H_{i}\right)^{T} v_{i}+\gamma_{p}\left(-\lambda v_{i}+\eta_{i}+d \zeta_{i}\right) \prec 0 .
$$

It follows that

$$
\left(\sum_{p=1}^{n} \gamma_{p} \widetilde{A}_{i}^{p}+\sum_{p=1}^{n} \gamma_{p} F_{i} H_{i}\right)^{T} v_{i}+\sum_{p=1}^{n} \gamma_{p}\left(-\lambda v_{i}+\eta_{i}+d \zeta_{i}\right) \prec 0 .
$$

Note that $\sum_{p=1}^{n} \gamma_{p}=1$ and $\widetilde{A}_{i}=\sum_{p=1}^{n} \gamma_{p} \widetilde{A}_{i}^{p}$; then

$$
\left(\widetilde{A}_{i}+F_{i} H_{i}\right)^{T} v_{i}+\left(-\lambda v_{i}+\eta_{i}+d \zeta_{i}\right) \prec 0 .
$$

Similarly, we get

$$
\widetilde{B}_{i}^{T} v_{i}-(1-\rho) \eta_{i}<0 .
$$

Applying (40) and (41) to (37) yields

$$
\dot{V}_{i}(t)-\lambda V_{i}(t)<w^{T}(t) G_{i}^{T} v_{i} .
$$

The remainder of the proof is similar to (23)-(31) and omitted. Therefore the system (3) is finite-time bounded under the designed controller. This completes the proof of Theorem 14.

Remark 15. Inequalities (11), (13), (32), and (34) are LMI. Once inequality (11) or (32) is resolved, the values of $\xi_{i}$ and $v_{i}$ are obtained. Substituting $\xi_{i, j}$ and $v_{i}$ into $\xi_{i}=\left(F_{i} H_{i}\right)^{T} v_{i}, H_{i}$ is obtained. Furthermore, if inequality (13) or (34) is satisfied, then $H_{i}$ is the desired gain matrix. By this way, the desired controller is built such that system (3) is finite-time bounded.

\section{Numerical Example}

A numerical example is given to illustrate the validities of the obtained results in this section. Consider the following system with interval uncertainties:

$$
\begin{gathered}
\dot{x}(t)=\left(A_{\sigma(t)}+\Delta A_{\sigma(t)}\right) x(t)+\left(B_{\sigma(t)}+\Delta B_{\sigma(t)}\right) x(t-h(t)) \\
+F_{\sigma(t)} u(t)+G_{\sigma(t)} w(t), \\
x(t)=\varphi(t) \quad t \in[-d, 0),
\end{gathered}
$$

where

$$
\begin{aligned}
& A_{1}=\left[\begin{array}{cc}
-7 & 7 \\
5 & -7
\end{array}\right], \quad B_{1}=\left[\begin{array}{ll}
0.2 & 0.9 \\
0.1 & 0.3
\end{array}\right], \\
& F_{1}=\left[\begin{array}{cc}
1 & 0 \\
0 & 0.5
\end{array}\right], \quad G_{1}=\left[\begin{array}{cc}
0.2 & 0.1 \\
0.1 & 0.15
\end{array}\right] \text {, } \\
& A_{2}=\left[\begin{array}{cc}
-7 & 7 \\
3 & -6
\end{array}\right], \quad B_{2}=\left[\begin{array}{cc}
0.2 & 0.1 \\
1 & 0.4
\end{array}\right] \text {, } \\
& F_{2}=\left[\begin{array}{cc}
0.5 & 0 \\
0 & 1
\end{array}\right], \quad G_{2}=\left[\begin{array}{cc}
0.2 & 0.1 \\
0.1 & 0.15
\end{array}\right] \text {, } \\
& \bar{A}_{1}=\left[\begin{array}{cc}
-6 & 8 \\
6 & -6
\end{array}\right], \quad \underline{A}_{1}=\left[\begin{array}{cc}
-8 & 6 \\
4 & -8
\end{array}\right] \text {, } \\
& \bar{A}_{2}=\left[\begin{array}{cc}
-6 & 8 \\
4 & -5
\end{array}\right], \quad \underline{A}_{2}=\left[\begin{array}{cc}
-8 & 6 \\
2 & -7
\end{array}\right] \text {, } \\
& \bar{B}_{1}=\left[\begin{array}{cc}
0.3 & 1 \\
0.2 & 0.4
\end{array}\right], \quad \underline{B}_{1}=\left[\begin{array}{cc}
0.1 & 0.8 \\
0 & 0.2
\end{array}\right] \text {, } \\
& \bar{B}_{2}=\left[\begin{array}{ll}
0.3 & 0.2 \\
1.1 & 0.5
\end{array}\right], \quad \underline{B}_{2}=\left[\begin{array}{cc}
0.1 & 0 \\
0.9 & 0.3
\end{array}\right], \\
& h(t)=0.1 t, \quad \rho=0.1, \quad T_{f}=10, \quad d=1, \\
& \varphi(t)=\left[\begin{array}{ll}
55 & 55
\end{array}\right]^{T}, \quad \omega^{T}(t)=\left[\begin{array}{ll}
\sin (t)+1 & 2 \cos (t)
\end{array}\right], \\
& n=2, \quad \gamma=2 \text {. }
\end{aligned}
$$

Obviously, $C_{1}=110$. Specify $\lambda=0.1, \beta=1.05, C_{2}=200$. Substituting these known parameters into (11) and (12) leads to feasible solutions that

$$
\begin{aligned}
& v_{1}=\left[\begin{array}{ll}
15.475 & 25.888
\end{array}\right]^{T}, \quad v_{2}=\left[\begin{array}{ll}
27.692 & 13.415
\end{array}\right]^{T}, \\
& \eta_{1}=\left[\begin{array}{ll}
40.366 & 48.327
\end{array}\right]^{T}, \quad \eta_{2}=\left[\begin{array}{ll}
46.951 & 41.572
\end{array}\right]^{T}, \\
& \zeta_{1}=\left[\begin{array}{ll}
33.802 & 33.802
\end{array}\right]^{T}, \quad \zeta_{2}=\left[\begin{array}{ll}
33.802 & 33.802
\end{array}\right]^{T}, \\
& \xi_{1}=\left[\begin{array}{ll}
-168.9 & -81.818
\end{array}\right]^{T}, \quad \xi_{2}=\left[\begin{array}{lll}
0.71057 & -262.3
\end{array}\right]^{T} \text {. }
\end{aligned}
$$

Then, it follows that

$$
\begin{gathered}
K_{0}=0.2781, \quad K_{1}=27.692, \\
K_{2}=48.327, \quad K_{3}=33.802, \\
K_{4}=13.415 .
\end{gathered}
$$


Noting that $\xi_{i}=\left(F_{i} H_{i}\right)^{T} v_{i}$, we obtain

$$
\begin{aligned}
H_{1}= & {\left[\begin{array}{cc}
-2.1843 & 0 \\
0 & 2.6114
\end{array}\right], \quad H_{2}=\left[\begin{array}{cc}
2.4413 & 0 \\
0 & 2.5197
\end{array}\right], } \\
& \underline{A}_{1}+F_{1} H_{1}=\left[\begin{array}{cc}
-5.8157 & 6 \\
4 & -6.6943
\end{array}\right] \in M, \\
& \underline{A}_{2}+F_{2} H_{2}=\left[\begin{array}{cc}
-6.77935 & 6 \\
2 & -4.4803
\end{array}\right] \in M .
\end{aligned}
$$

Consequently, $H_{1}$ and $H_{2}$ meet the requirements of the design. Then the controller $u(t)=H_{\sigma(t)} x(t)$ is obtained.

On the other hand, from (14), we get

$$
\tau_{a}>0.5469
$$

According to Theorem 10, if we choose a $\sigma(t)$ which satisfies $\tau_{a}>0.5469$, then system (43) would be finitetime bounded with respect to $\left(C_{1}, C_{2}, T_{f}, \gamma, \sigma(t)\right)$ under the designed controller $u(t)=H_{\sigma(t)} x(t)$.

Finally, the simulation of system (43) with the designed controller is performed. The simulation results are shown in Figures 1-3. The switching law of system (43) is shown in Figure 1. Obviously, the switching times of system (43) is 7 over $[0,10]$. Thus, $\tau_{a}=(10 / 7)>0.5469$ and meets the requirement of (14). In Figure 2, we could find that every element of $x(t)$ falls in the range $[0,100]$. Therefore, every element of system state is bounded which implies that the system state is bounded over $[0,10]$. In other words, system (43) is FTB. The same conclusion could be reached via Figure 3. From Figure 3, it is easy to find $\|x\|<C_{2}=200$ over $[0,10]$. Thus, the state of system (43) remains within the prescribed bound over $[0,10]$. Therefore, under the designed controller, system (43) is indeed bounded over $[0,10]$.

In addition, though system (43) is finite-time bounded, its state trajectory is an oscillating curve over $[0,10]$. Thus, system (43) is not Lyapunov stable over $[0,10]$. It is illustrated that FTB is different from LAS. There is not a necessary relation between them.

\section{Conclusions}

This paper focuses on the FTS analysis for a class of uncertain switched positive linear systems. Based on multiply copositive Lyapunov-Krasovskii functions and average dwell-time method, a robust state feedback controller is built such that the uncertain system is finite-time bounded. The innovation points lie in that a concise definition of FTB is proposed for switched positive system. Since the new definition is adopted, the subsequent mathematical derivation is greatly simplified. Finally, a numerical example illustrates the validities of the obtained results and the difference between LAS and FTB.

However, multiply copositive Lyapunov-Krasovskii functions in this paper are traditional. In the literature [32, 33], barrier Lyapunov functions and P-times differentiable unbounded functions are presented, respectively. In future work, we will try to utilize the two new kinds of functions to deal with the FTS problem for switched positive system and

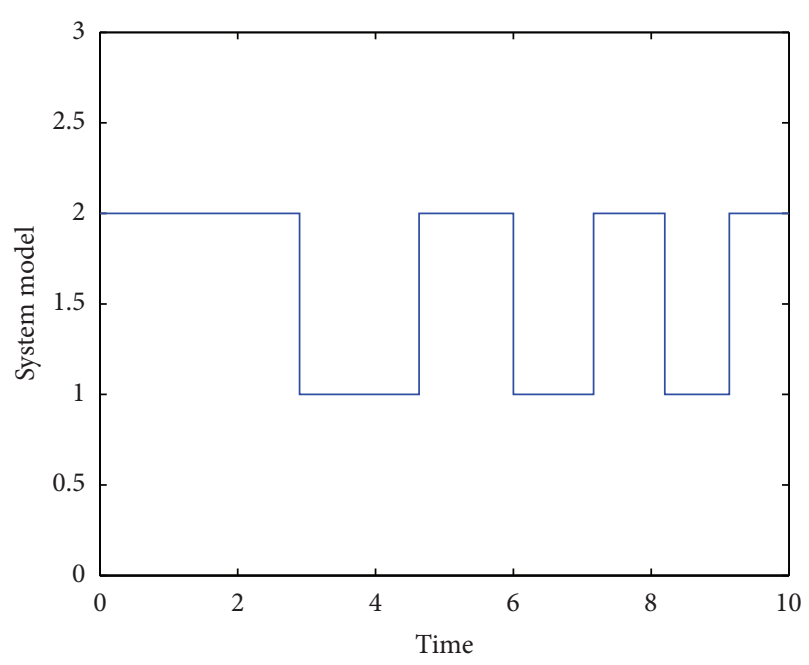

FIGURE 1: The figure of switching law.

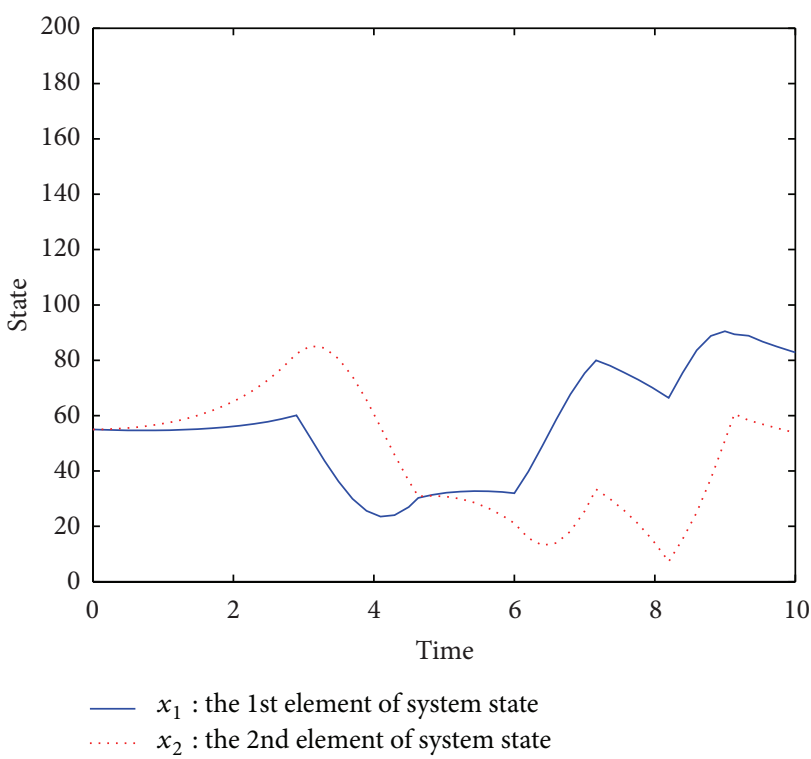

FIGURE 2: The figure of system state.

make a comparative analysis with the traditional functions. In addition, interval and polytopic uncertainties could not cover all kinds of the uncertainties in practice. Therefore, we will analyze the problem of FTS for switched positive linear system with arbitrary uncertainties. As mentioned in Section 1, the switching behavior is an important factor for the problems of FTB and FTS. For FTB and FTS, it is one of the critical issues to analyze asynchronous switching [34], slow switching, and fast switching impacts on the system state. In this paper, the issue has not been discussed in detail. Therefore we will try to investigate this issue for switched positive system in further study. 


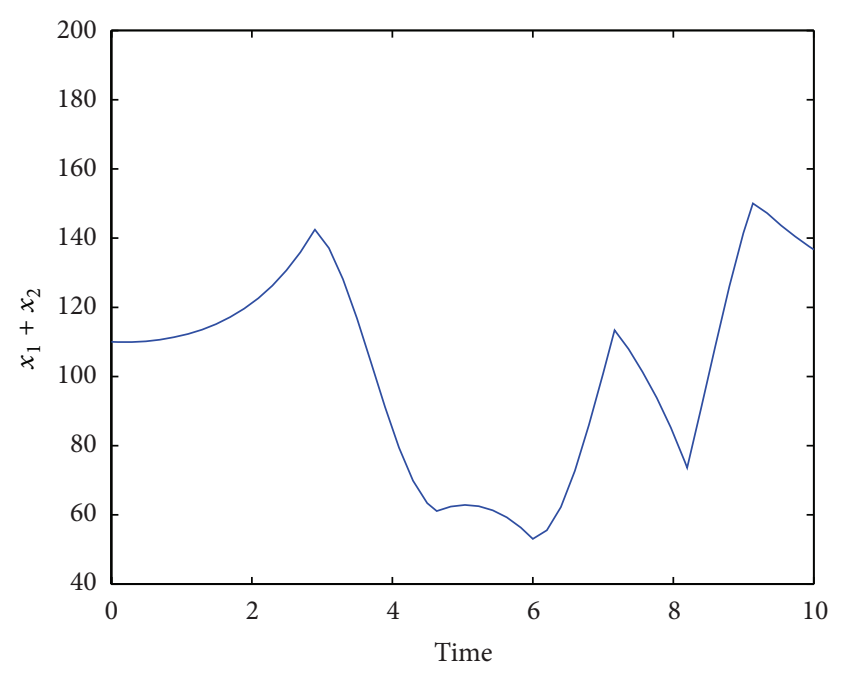

FIGURE 3: The figure of $\|x\|$.

\section{Conflict of Interests}

The authors (Tianjian Yu, Yanke Zhong, Tefang Chen, and Chunyang Chen) declare that there is not any conflict of interests regarding the publication of this paper.

\section{Acknowledgment}

This work was supported by the National Natural Science Foundation of China under Grant no. 61273158.

\section{References}

[1] D. Liberzon, Switching in Systems and Control, Springer, Boston, Mass, USA, 2003.

[2] Y. Zhong and T. Chen, "Robust observer design for switched positive linear system with uncertainties," Abstract and Applied Analysis, vol. 2014, Article ID 745906, 9 pages, 2014.

[3] A. Jadbabaie, J. Lin, and A. S. Morse, "Coordination of groups of mobile autonomous agents using nearest neighbor rules," IEEE Transactions on Automatic Control, vol. 48, no. 6, pp. 988-1001, 2003.

[4] R. Shorten, F. Wirth, and D. Leith, "A positive systems model of TCP-like congestion control: asymptotic results," IEEE/ACM Transactions on Networking, vol. 14, no. 3, pp. 616-629, 2006.

[5] E. Hernandez-Vargas, P. Colaneri, R. Middleton, and F. Blanchini, "Discrete-time control for switched positive systems with application to mitigating viral escape," International Journal of Robust and Nonlinear Control, vol. 21, no. 10, pp. 1093-1111, 2011.

[6] Y. O. Lee, Y. S. Son, and C. C. Chung, "Clamping force control for an electric parking brake system: switched system approach," IEEE Transactions on Vehicular Technology, vol. 62, no. 7, pp. 2937-2948, 2013.

[7] L. Gurvits, R. Shorten, and O. Mason, "On the stability of switched positive linear systems," IEEE Transactions on Automatic Control, vol. 52, no. 6, pp. 1099-1103, 2007.

[8] X. Xue and Z. Li, "Asymptotic stability analysis of a kind of switched positive linear discrete systems," IEEE Transactions on Automatic Control, vol. 55, no. 9, pp. 2198-2203, 2010.
[9] W. Xiang, J. Xiao, and I. M. Naveed, "Asymptotic stability, L2 gain boundness analysis and control synthesis for switched systems: a switching frequency approach," International Journal of Adaptive Control and Signal Processing, vol. 26, no. 4, pp. 350373, 2012.

[10] W. Xiang and J. Xiao, "Stability analysis and control synthesis of switched impulsive systems," International Journal of Robust and Nonlinear Control, vol. 22, no. 13, pp. 1440-1459, 2012.

[11] X. Zhao, L. Zhang, P. Shi, and M. Liu, "Stability of switched positive linear systems with average dwell time switching," Automatica, vol. 48, no. 6, pp. 1132-1137, 2012.

[12] X. Zhao, X. Liu, S. Yin, and H. Li, "Improved results on stability of continuous-time switched positive linear systems," Automatica, vol. 50, no. 2, pp. 614-621, 2014.

[13] A. Benzaouia and F. Tadeo, "Stabilization of positive switching linear discrete-time systems," International Journal of Innovative Computing, Information and Control, vol. 6, no. 6, pp. 24272437, 2010.

[14] Y. Sun, "Stabilization of switched systems with nonlinear impulse effects and disturbances," IEEE Transactions on Automatic Control, vol. 56, no. 11, pp. 2739-2743, 2011.

[15] W. Xiang and J. Xiao, "Stabilization of switched continuoustime systems with all modes unstable via dwell time switching," Automatica, vol. 50, no. 3, pp. 940-945, 2014.

[16] X. Lin, S. Li, and Y. Zou, "Finite-time stability of switched linear systems with subsystems which are not finite-time stable," IET Control Theory \& Applications, vol. 8, no. 12, pp. 1137-1146, 2014.

[17] S. Mastellone, C. T. Abdallah, and P. Dorato, "Stability and finite time stability of discrete-time nonlinear networked control systems," in Proceedings of the American Control Conference (ACC '05), pp. 1239-1244, June 2005.

[18] L. Wang, L. Cai, X. Liu, and X. Shen, "Practical stability and bounds of heterogeneous AIMD/RED system with time delay," in Proceedings of the IEEE International Conference on Communications (ICC '08), pp. 5558-5563, May 2008.

[19] X. Lin, H. Du, and S. Li, "Finite-time boundedness and $L_{2}$ gain analysis for switched delay systems with norm-bounded disturbance," Applied Mathematics and Computation, vol. 217, no. 12, pp. 5982-5993, 2011.

[20] M. Xiang and Z. Xiang, "Finite-time $L_{1}$ control for positive switched linear systems with time-varying delay," Communications in Nonlinear Science and Numerical Simulation, vol. 18, no. 11, pp. 3158-3166, 2013.

[21] H. Liu, Y. Shen, and X. Zhao, "Finite-time stabilization and boundedness of switched linear system under state-dependent switching," Journal of the Franklin Institute, vol. 350, no. 3, pp. 541-555, 2013.

[22] Y. Zhong and T. Chen, "Finite-time boundedness analysis for a class of switched linear systems with time-varying delay," Abstract and Applied Analysis, vol. 2014, Article ID 982414, 9 pages, 2014.

[23] W. Xiang and J. Xiao, " $H_{\infty}$ finite-time control for switched nonlinear discrete-time systems with norm-bounded disturbance," Journal of the Franklin Institute, vol. 348, no. 2, pp. 331-352, 2011.

[24] X. Liu, D. W. C. Ho, W. Yu, and J. Cao, "A new switching design to finite-time stabilization of nonlinear systems with applications to neural networks," Neural Networks, vol. 57, pp. 94-102, 2014.

[25] I. M. Naveed, J. Xiao, and W. Xiang, "Finite-time H-infinity sate estimation for discrete-time switched control systems under asynchronous switching," Asian Journal of Control, vol. 16, no. 4, pp. 1112-1121, 2014. 
[26] G. Chen and Y. Yang, "Finite-time stability of switched positive linear systems," International Journal of Robust and Nonlinear Control, vol. 24, no. 1, pp. 179-190, 2014.

[27] G. Chen, Y. Yang, and Q. Pan, "Finite time stability analysis of switched systems with stable and unstable subsystems," Asian Journal of Control, vol. 16, no. 4, pp. 1224-1228, 2014.

[28] J. Cheng, H. Zhu, S. Zhong, F. Zheng, and K. Shi, "Finitetime boundedness of a class of discrete-time Markovian jump systems with piecewise-constant transition probabilities subject to average dwell time switching," Canadian Journal of Physics, vol. 92, no. 2, pp. 93-102, 2014.

[29] Y. Wang, X. Shi, G. Wang, and Z. Zuo, "Finite-time stability for continuous-time switched systems in the presence of impulse effects," IET Control Theory \& Applications, vol. 6, no. 11, pp. 1741-1744, 2012.

[30] L. Weiss and E. F. Infante, "Finite time stability under perturbing forces and on product spaces," IEEE Transactions on Automatic Control, vol. 12, pp. 54-59, 1967.

[31] P. Dorato, "An overview of finite-time stability," in Current Trends in Nonlinear Systems and Control, pp. 185-194, Birkhauser, Boston, Mass, USA, 2006.

[32] B. Niu and J. Zhao, "Barrier Lyapunov functions for the output tracking control of constrained nonlinear switched systems," Systems and Control Letters, vol. 62, no. 10, pp. 963-971, 2013.

[33] N. Ben and J. Zhao, "P-times differentiable unbounded functions for roust control of uncertain nonlinear switched systems with tracking constraints," International Journal of Robust and Nonlinear Control, 2014.

[34] W. Xiang, J. Xiao, and M. N. Iqbal, "Robust observer design for nonlinear uncertain switched systems under asynchronous switching," Nonlinear Analysis: Hybrid Systems, vol. 6, no. 1, pp. 754-773, 2012. 


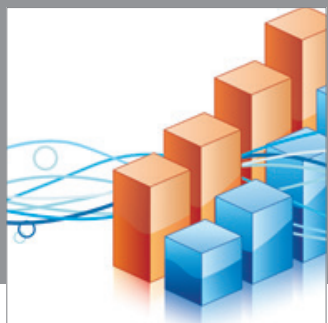

Advances in

Operations Research

mansans

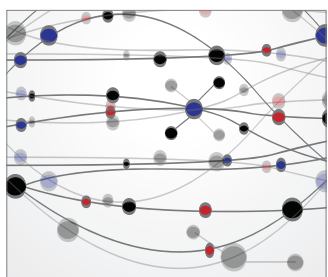

The Scientific World Journal
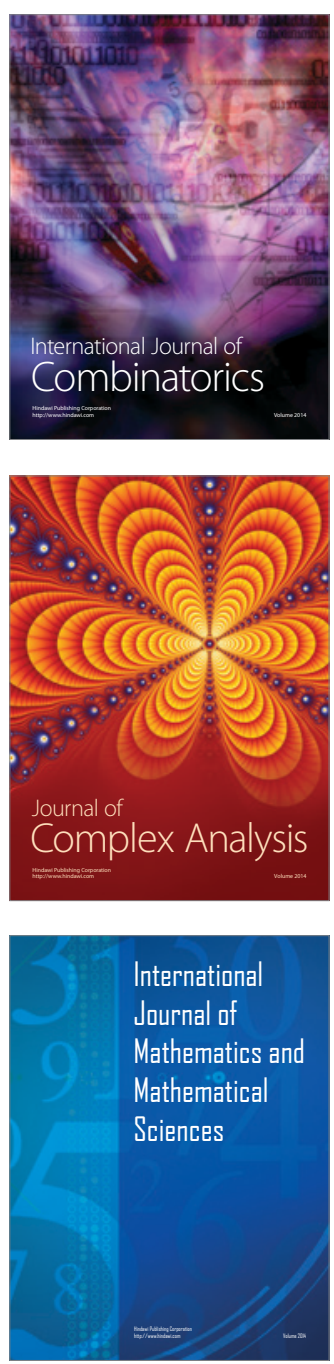
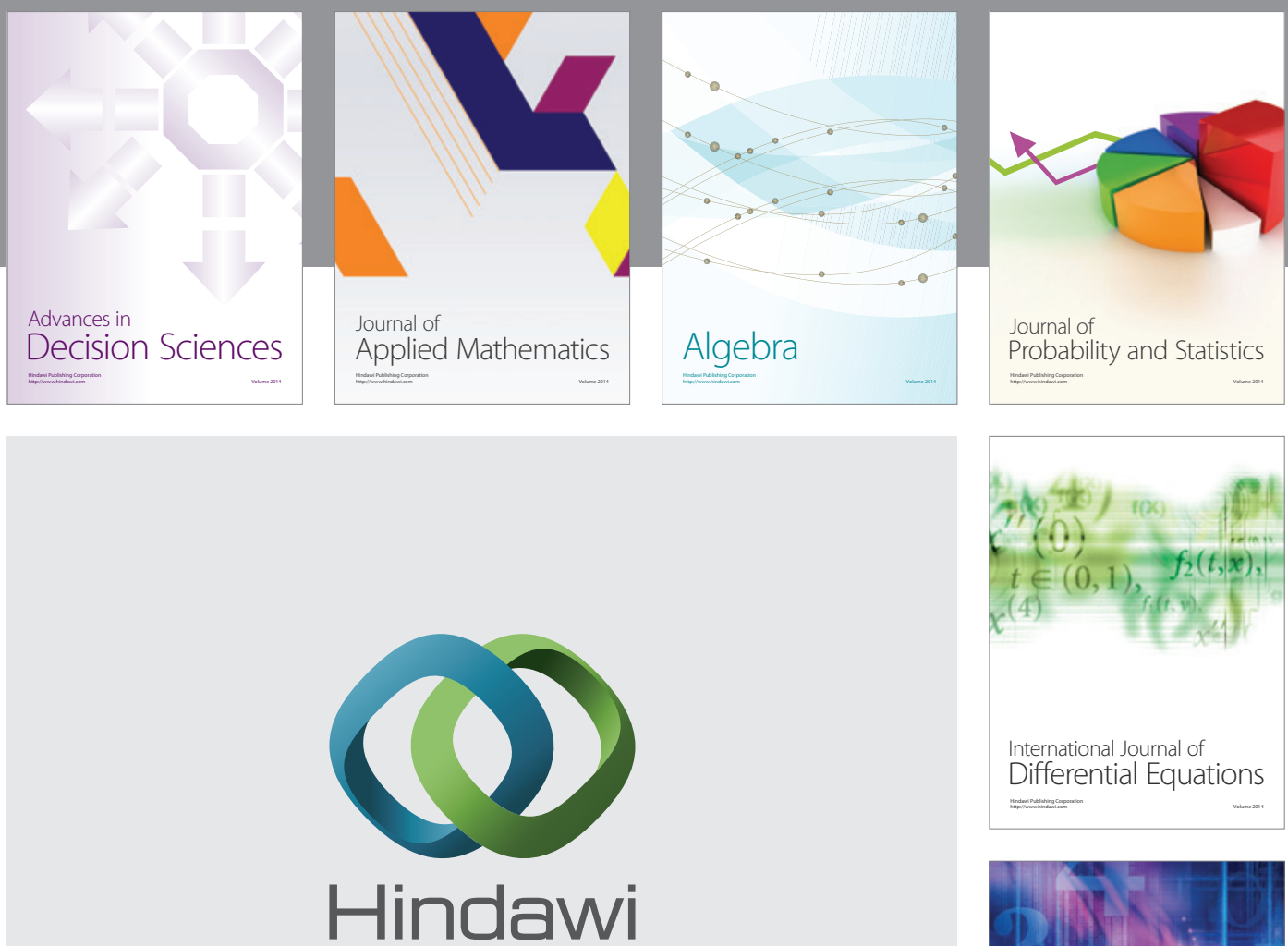

Submit your manuscripts at http://www.hindawi.com
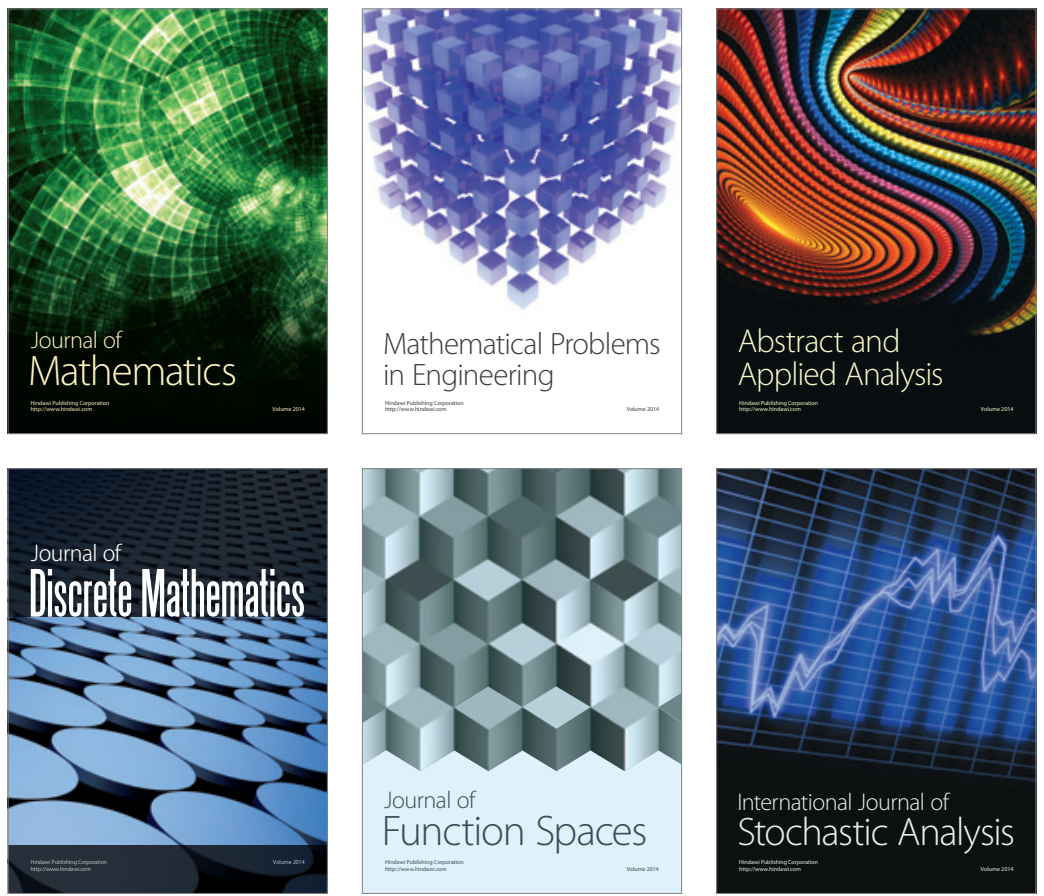

Journal of

Function Spaces

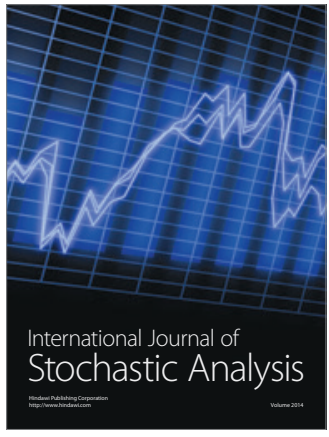

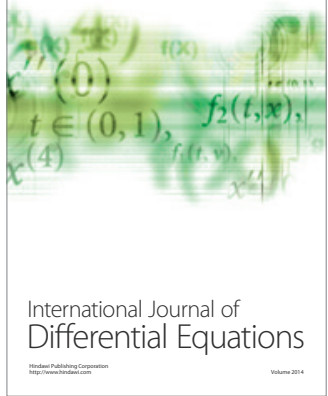
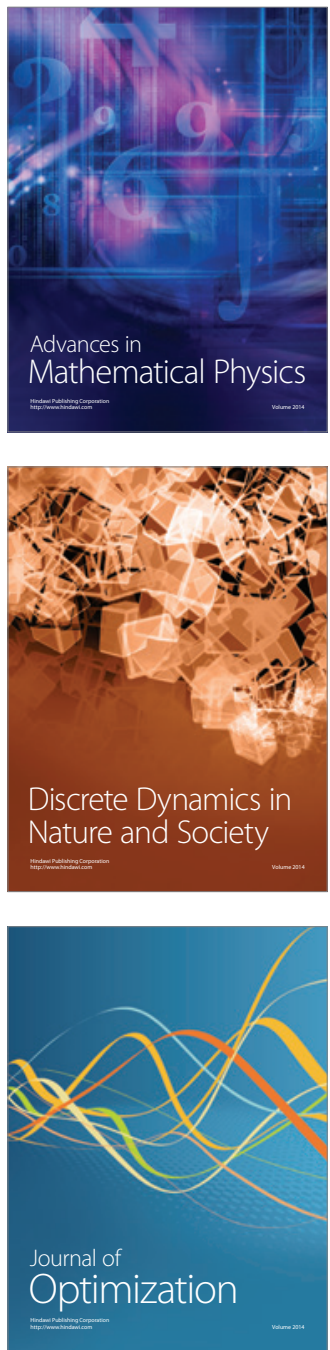